\section{APLICACIÓN DEL MÉTODO MORA-VAHRSON PARA EVALUAR LA SUSCEPTIBILIDAD A DESLIZAMIENTO EN EL MUNICIPIO DE MANAURE, CESAR, COLOMBIA}

Ruth E. López ${ }^{1}$, Amat D. Zuluaga ${ }^{1 *}$, Felipe Gómez ${ }^{2}$ y Luis Tapia ${ }^{1}$

\section{RESUMEN}

La determinación de las zonas de amenazas es el punto de partida en la gestión de riesgos por deslizamiento, para esto existe una serie de modelos que permiten establecer las zonas más susceptibles a presentar movimientos de remoción en masa, los cuales representan un peligro para la población aledaña. El método Mora-Vahrson fue usado para la caracterización el grado de susceptibilidad de las unidades geológicas presentes al este del municipio de Manaure, en el Departamento del Cesar, Colombia. Con ello, se identificaron posibles escenarios que puedan asociar riesgos geológicos relacionados a los procesos morfodinámicos que presentan dichas unidades. Se evaluó la susceptibilidad para dos escenarios posibles en el área de estudio: el primer escenario presenta la figura activa de la falla Manaure (situación actual) y un segundo presenta la reactivación de esta. En la primera situación, más del $70 \%$ de la zona presenta un grado de susceptibilidad medio, mientras que la zona de montaña hacia el oeste presenta en ciertas partes grados altos de susceptibilidad. En el segundo escenario, los índices de susceptibilidad aumentan de manera exponencial, con grados de alto en el $90 \%$ del área, mientras que el $10 \%$ restante con grado medio.

\section{PALABRAS CLAVE}

Mora-Vahrson, Gestión del riesgo, Susceptibilidad, Riesgo de desastre, Colombia

\section{APPLICATION OF THE MORA-VAHRSON METHOD TO ASSESS LANDSLIDE SUSCEPTIBILITY IN THE MUNICIPALITY OF MANAURE, CESAR, COLOMBIA}

\section{ABSTRACT}

Assessing hazardous areas is the starting point in landslide risk management. To that end, there are a series of models that allow establishing the most susceptible areas to mass movements, which represent hazards and risks to the population nearby. The Mora-Vahrson method is utilized in this study to assess the degree of landslide susceptibility of the geological units at the east of the Municipality of Manaure, in the Department of Cesar, Colombia. Additionally, possible scenarios that could be associated to geological risks of morphodynamic processes were identified. Landslide susceptibility was evaluated for two scenarios in the study area: The first scenario bases on the active figure of the Manaure fault (current situation), while the second bases on its reactivation. In the first scenario, more than 70 percent of the area has a 'medium' degree of susceptibility. Certain locations in the mountainous area obtained 'high' degrees of susceptibility. In the second scenario, susceptibility degrees increased exponentially, with 'high' levels in 90 percent of the study area, while the remaining 10 percent obtained 'medium'.

\section{KEYWORDS}

Mora-Vahrson, Risk management, Susceptibility, Disaster risk, Colombia
1. Grupo de Investigación en Ingeniería Geológica GIINGEO, Fundación Universitaria del Área Andina, Valledupar, Colombia.

2. Oficina Departamental para la Gestión del Riesgo de Desastres ODGRD, Gobernación del Departamento del Cesar, Valledupar, Colombia.

*Autor de correspondencia: azuluagaı6@areandina.edu.co

\section{RECIBIDO}

27 de septiembre de 2019

\section{ACEPTADO}

27 de noviembre de 2019

\section{PUBLICADO}

1 de julio de 2020

\section{Formato cita}

Recomendada (APA): López, R.E., Zuluaga, A.D., Gómez, F. \& Tapia, L. (2020). Aplicación del Método Mora-Vahrson para Evaluar la Susceptibilidad a Deslizamiento en el Municipio de Manaure, Cesar, Colombia. Revista de Estudios Latinoamericanos sobre Reducción del Riesgo de Desastres REDER, 4(2), 57-70.

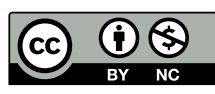

Todos los artículos publicados en REDER siguen una política de Acceso Abierto y se respaldan en una Licencia CreativeCommons Atribución-NoComercial 4.0 Internacional.

Revista de Estudios Latinoamericanos sobre Reducción del Riesgo de Desastres (REDER)

Diseño: Lupe Bezzina Tipografía: Hospital 


\section{INTRODUCCIÓN}

En Colombia, país que por sus condiciones ecosistemáticas contiene gran variedad de características físicas y una geodinámica que condiciona el advenimiento de amenazas naturales de todo tipo, se están implementando nuevas metodologías que ayudan al control y supervisión de eventos naturales que puedan ser considerados de alto riesgo, para disminuir la vulnerabilidad de los ecosistemas que comparten estos escenarios. Los procesos geológicos y climáticos que afectan a la superficie terrestre crean el relieve y definen la morfología de las laderas, que va modificándose a lo largo del tiempo para adaptarse a nuevas condiciones geológicas o climáticas (González et al., 2002). En el norte colombiano, específicamente en el Departamento del Cesar, se encuentran diferentes accidentes geográficos como la Sierra Sevada de Santa Marta, la serranía del Perijá y otras formaciones. Este departamento es una de las principales zonas de Colombia en las cuales los indicadores de riesgo están en alerta constante. Según Acosta y Rodríguez (2016), el municipio de Manaure presenta doce escenarios de riesgo de origen geológico, de los cuales, once están asociados a procesos de remoción en masa, y uno general de sismos. Esto ha despertado la alerta en los estudiosos del tema, para poder implementar estrategias que proporcionen acciones preventivas o correctivas encaminadas a la mitigación de los efectos causados por estas amenazas naturales. Entre estas estrategias, se encuentra la caracterización del grado de susceptibilidad presente en las unidades geológicas para determinar las áreas más susceptibles a presentar procesos de remoción en masa y de esta manera intuir futuros eventos de movimientos de masa.

El Departamento del Cesar se encuentra divido en seis regiones naturales, la Sierra Nevada de Santa Marta (SNSM), la serranía del Perijá, el complejo cenagoso de la Zapatoza y los valles de los ríos Cesar, Ariguaní y Magdalena (Gobernación del Cesar, 2019). Manaure está ubicado en la vertiente occidental de la serranía del Perijá a $775 \mathrm{msnm}$. Dista de Valledupar en $34 \mathrm{~km}$ y las coordenadas geográficas correspondientes a Manaure son: $10^{\circ} 24^{\prime \prime} \mathrm{N}$ y 73010" W.

En síntesis, este documento de investigación aplica el método Mora-Vahrson (1994) para la caracterización de escenarios susceptibles a movimientos en masa de las unidades geológicas de estudio y utiliza información de campo y mapas sísmicos y pluviométricos del municipio de Manaure para zonificar las áreas más propensas a eventos de deslizamientos.

\section{LOCALIZACION}

El área de estudio se encuentra en las estribaciones de la serranía de Perijá, al este del Departamento del Cesar, entre el municipio de Manaure y la vereda Hondo del río (ver Figura 1), presenta un clima templado debido a los tres pisos térmicos que este municipio presenta, la temperatura promedio es de $24^{\circ} \mathrm{C}$. El estudio se realizó sobre la plancha 27 del Instituto Geográfico Agustín Codazzi (IGAC, 2019), un área de $12 \mathrm{~km}^{2}$, con las siguientes coordenadas representadas en la Tabla 1.

\begin{tabular}{lll} 
Punto & $X$ & $Y$ \\
\hline 1 & 1115000 & 1642500 \\
\hline 2 & 1120000 & 1642500 \\
\hline 3 & 1115000 & 1640000 \\
\hline 4 & 1120000 & 1640000 \\
\hline
\end{tabular}

Tabla 1. Coordenadas del área de estudio

Fuente: Autores, 2020

\section{ANTECEDENTES}

Colombia enfrenta grandes retos que amenazan seriamente su desarrollo. Factores como el desplazamiento en las zonas rurales a las zonas urbanas, la degradación ambiental y el cambio acelerado del uso del suelo amplifican dichos retos. Estas condiciones socio-económicas, asociadas a la propensión del país a la ocurrencia de fenómenos naturales, tales como sismos, inundaciones y deslizamientos, entre otros, exacerbados por las acciones humanas y las condiciones variantes del clima, confirman un proceso continuo de construcción y acumulación de riesgos. La materialización de estos escenarios amenazantes se convierte en lo que se denomina un desastre, afectando el desarrollo del país, impidiendo y retrasando el logro de las metas de bienestar social trazadas por el Gobierno (Campos, 2012). Diferentes autores han advertido de la importancia de las evaluaciones de amenaza y susceptibilidad por fenómenos naturales (Hidalgo \& Vega, 2014; Mora, 2004; Quesada \& Feoli, 2018; Ramirez, 2006; Suares, 1998; Vivanco, 2011), sin embargo, Colombia 
en los planes de ordenamiento territorial de los municipios aún no generan estudios de amenazas confiables, que permitan realmente una planificación consciente de los municipios y las estrategias de acción ante los fenómenos naturales.

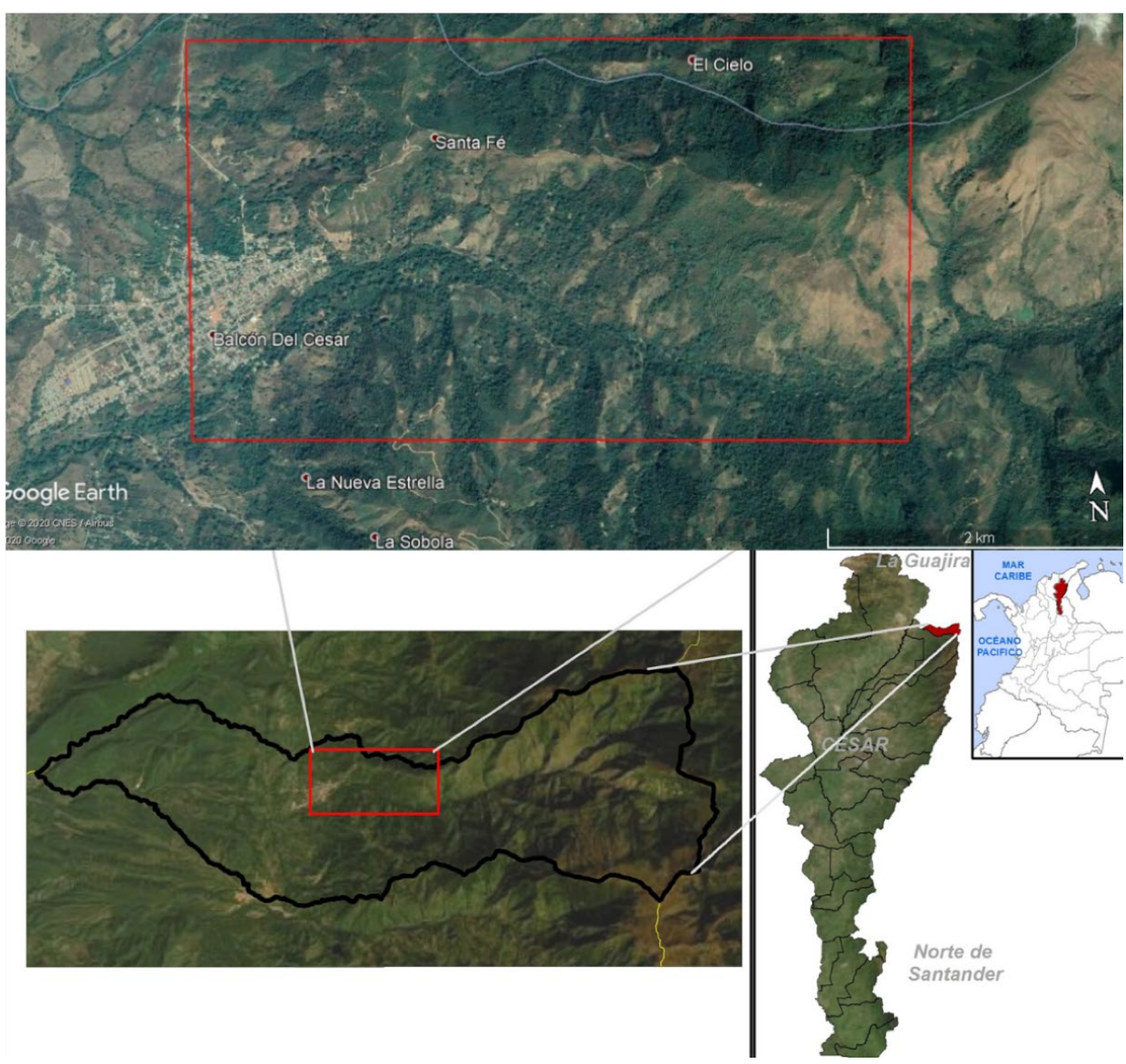

Figura 1. Mapa zona de estudio

Fuente: Autores, 2020, basado en imágenes de Google Earth Pro v7.3

Como consecuencia de las grandes pérdidas sufridas por la ocurrencia del fenómeno de La Niña 2010 - 2011, y en el ámbito de la agenda que en materia de gestión del riesgo de desastres que el Banco Mundial ha mantenido desde 1999 con el Gobierno colombiano, el Departamento Nacional de Planeación solicitó el apoyo de la institución para llevar a cabo una evaluación integral de las políticas de gestión del riesgo, así como en la formulación de recomendaciones estratégicas a corto y largo plazo, que contribuyan a reducir la afectación de la población y el impacto económico de los desastres (Campos, 2012). El Cambio Climático ha sido considerado un problema inevitable con consecuencias muy graves para todo el planeta, y en el 2016 el Departamento del Cesar se declaró en calamidad pública por temporada seca en el $80 \%$ del territorio, lo que involucró al total de su población (1.028.890 hab.) y por ende al Municipio de Manaure, objeto de esta investigación (Acosta \& Rodriguez, 2016).

Por el lado de amenaza por movimientos en masa, la mayor exposición se identifica en 353 municipios que se localizan en zonas de amenaza alta y muy alta, y en los cuales se concentra el $8 \%$ de la población nacional. En exposición moderada se encuentran 346 municipios, que corresponden al 23\% de la población. Los departamentos que se destacan por tener mayor población en zonas de amenaza muy alta y alta son Antioquia, Cundinamarca, Cauca, Santander, Boyacá, Caldas y Tolima, seguidos por Norte de Santander, Nariño, Huila, Risaralda, Cesar y Bolívar. A nivel municipal, la mayor cantidad de población expuesta a amenaza alta y muy alta por movimientos en masa está en los municipios de Villamaría (Caldas); Bolívar, La Vega, Piendamó y Cajibío (Cauca); Yarumal y Urrao (Antioquia); Líbano (Tolima); Santa Rosa del Sur (Bolívar); y San Vicente de Chucurí (Campos, 2012)

Una de las estrategias utilizadas para la prevención e identificación de amenazas de movimientos de remoción en masa, es la metodología Mora-Vahrson (1994) ya que esta permite 
realizar un análisis de las áreas extensas bajo amenaza de deslizamientos, utilizando indicadores morfodinámicos, tales como el relieve relativo, la litología, la humedad del suelo, la sismicidad y la lluvia, todo esto definido por índices de influencia para cada área, que pueden ser trabajados en un Sistemas de Información Geográfico (SIG), para obtener un mapa con potencial de deslizamientos (Barrantes et al., 2011).

En el municipio de Manaure las primeras señales de movimientos en el terreno están dadas a lo largo de grietas superficiales por medio de las cuales se pueden desencadenar deslizamientos (Gutierrez, 2008). Los índices de susceptibilidad por deslizamientos son de alta prioridad, ya que las unidades geológicas aledañas pueden generar escenarios de riesgos constantes para los pobladores del municipio, bien sea por las altas precipitaciones que se presentan o por la actividad sísmica de la zona, añadiendo el alto grado de meteorización que poseen. La litología circundante está constituida principalmente por rocas metamórficas de bajo grado de protolito sedimentario, filítas de grano fino y cuarcitas de coloraciones amarillentas de grano medio a fino, además también se encuentran calizas fosilíferas, areniscas de color rojizo, areniscas calcáreas, conglomerados: todas estas unidades presentan un grado medio-bajo de meteorización con excepción de algunas zonas de grado alto. Adicional a esto, el municipio es atravesado por la falla Manaure y muy cerca a esta han sido reportados movimientos telúricos. Por ello es indispensable determinar el comportamiento geomorfodinámico de las unidades geológicas de estudio, ya que, de esta manera se lograrán identificar las zonas puntuales de remoción en masa en base a los resultados que nos entregue el método Mora-Vahrson (1994) lo cual permitirá delimitar las áreas con alto potencial a los eventos de deslizamiento.

\section{METODOLOGIA}

La metodología Mora-Vahrson (1994) se utilizó con el objetivo de realizar mapas de zonificación de susceptibilidad a deslizamiento. Para obtener este tipo de mapas se tuvieron en cuenta los factores condicionantes y desencadenantes. Los factores condicionantes hacen referencia a la geología, geomorfología, grado de pendiente y cobertura vegetal, mientras que los factores desencadenantes hacen referencia a la sismicidad y las precipitaciones. Los análisis de estos factores se realizaron a una escala 1:25.000, para poder tener un mayor detalle de la información necesaria para la realización de cada uno de los mapas. Cabe resaltar que no hubo modificaciones a la metodología diferentes a los porcentajes de los factores condicionantes y desencadenantes evaluadas desde el trabajo en campo hasta los análisis de laboratorio.

Finalmente, con los mapas resultantes de los factores anteriormente mencionados, se realiza el mapa final de zonificación basándonos en la Ecuación 1.

\section{Ecuación 1.}

$$
S=F c+F d
$$

Donde:

S: Índice de susceptibilidad

Fc: Factores condicionantes

Fd: Factores desencadenantes

Teniendo en cuenta la ecuación anterior procedemos a determinar los factores condicionantes y desencadenantes, lo cual se determina con las siguientes ecuaciones:

Factores condicionantes:

\section{Ecuación 2.}

$$
F c=(\text { geo } * 0.3)+(\text { gmor } * 0.2)+(\text { cbve } * 0.2)+(\text { pen } * 0.3)
$$

Donde:

geo: Geología

gmor: Geomorfología

cbve: Cobertura

pen: Pendiente 
Factores desencadenantes:

\section{Ecuación 3.}

$$
F d=(\operatorname{sis} * 03)+(\text { pre } * 0.7)
$$

Donde:

sis: Sismicidad

pre: Precipitación

Para realización de los mapas correspondientes a los factores anteriormente mencionados, debemos tener en cuenta el porcentaje asignado por el método a cada parámetro (ver Tabla 2), teniendo en cuenta que el resultado de estos nos arroja el mapa final de zonificación.

\begin{tabular}{lll} 
Tipo & Parámetro & Porcentual (\%) \\
\hline \multirow{3}{*}{ Condicionantes } & Geología & 30 \\
\cline { 2 - 3 } & Geomorfología & 20 \\
\cline { 2 - 3 } & Pendiente & 30 \\
\cline { 2 - 3 } Desencadenantes & Cobertura vegetal & 20 \\
\hline \multirow{2}{*}{} & Precipitación & 70 \\
\cline { 2 - 3 } & Fallas geológicas & 30 \\
\hline
\end{tabular}

Tabla 2. Porcentaje de los parámetros

Fuente: Mora y Vahrson (1994)

\section{RESULTADOS}

Esta metodología por si misma resulta aplicable a países en vía de desarrollo, debido a que requieren pocas variables morfodinámicas y su aplicación es relativamente sencilla, incluye los factores más significativos desde el punto de vista de la inestabilidad de laderas, se basa en parámetros que pueden determinarse de manera ágil y económica. La metodología no sustituye los estudios geotécnicos de campo y laboratorio necesarios para la protección y mitigación correspondientes, ya que tampoco es capaz de pronosticar el tipo de deslizamiento que podría presentarse.

\section{Susceptibilidad por factores condicionantes.}

Los parámetros que corresponden a estos factores condicionante están constituidos de la siguiente manera:

Factor geológico. Corresponde a la geología presente en el área de estudio, que corresponden a las siguientes unidades (ver también Figura 2):

- Metasedimentitas cámbricas y ordovicias (EOms): Esta formación corresponde a rocas metamórficas, provenientes de un protolito sedimentaria, las cuales corresponden a filitas y cuarcitas de edad ordovícicas y cámbricas; las filitas presentan una textura de grano muy fino, brillo graso, con una coloración gris azulosa; por otro lado, las cuarcitas se encontraron moderadamente meteorizadas, con una tonalidad amarilla rojiza producto de las impurezas de los minerales.

- Sedimentitas devónicas y carboníferas de la cuchilla carbonal (Dcc): La litologia en esta formación es variada, en las sedimentitas devónicas se encontraron cuarcitas de grano medio a fino, sub-redondeados con baja porosidad de edad paleozoica superior, por otra parte, en las sedimentitas carboníferas se encontraron rocas calcareas fosilíferas en su mayoría presencia de brarquiópodos, estas rocas presentan una edad carbonífera superior

- Sedimentitas permicas (P): Se encuentran aflorando de base a techo en una secuencia de conglomerados sub-redondeados, de areniscas de color rojizo, seguidas de unas calizas de matriz arenosa y hacia el techo encontramos calizas fosilíferas, esta formación es de edad pérmica.

- $\quad$ Aluviones recientes (QAL): Se encuentran localizados en los valles, ríos, quebradas y mesetas. Debido a los flujos de los ríos que van transportado el material, y al perder energía en el flujo, va siendo depositado. Litológicamente este material se encuentra 
constituido por rocas sedimentarias, conglomerados, areniscas, limolitas, arcillolita, calizas y en ciertos sectores rocas de bajo grado de metamorfismo.

- Terrazas aluviales: Localizadas al Sur-Oriente de Manaure, litológicamente se encuentran Conformadas por gravas, cantos, arenas en una matriz areno- limosa, se encuentra en áreas pequeñas y escasamente cartografiables.

Teniendo en cuenta las unidades anterior mente mencionadas, se procede a realizar el mapa geológico (Figura 2), como primer factor condicionantes:
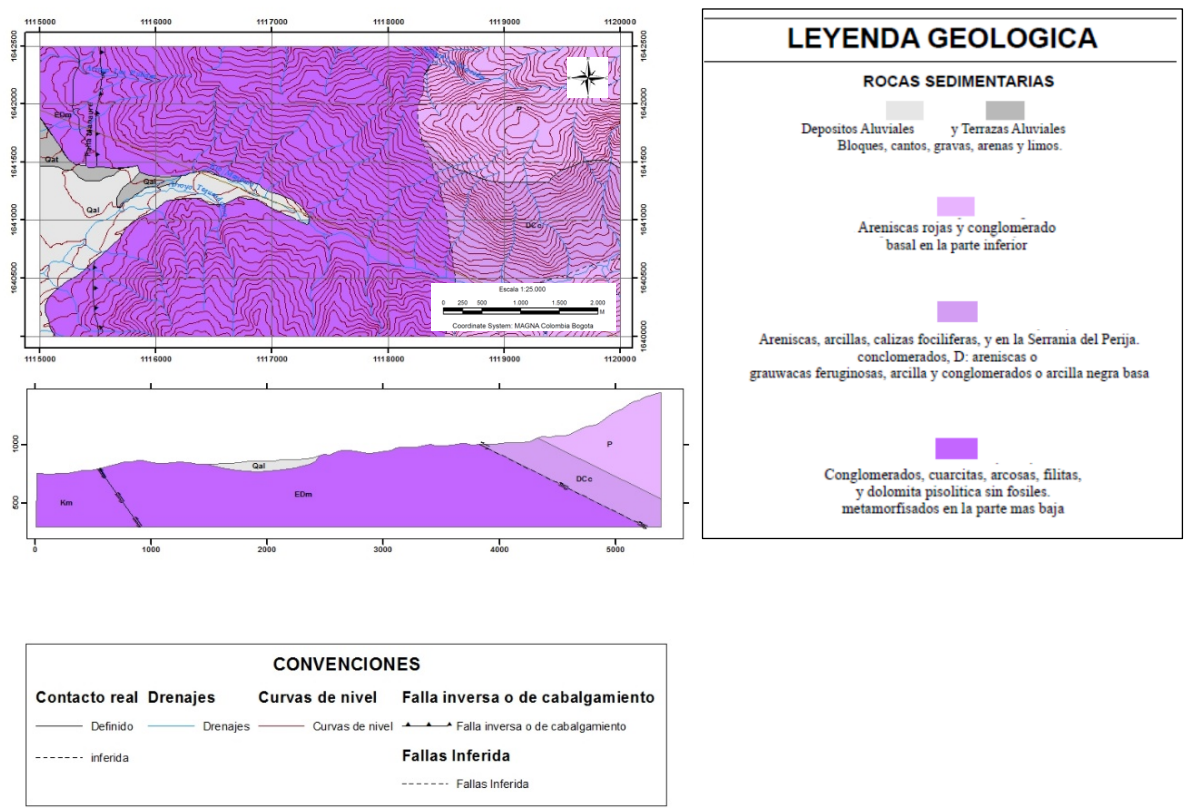

Figura 2. Mapa geológico de las unidades al este de Manaure Fuente: Autores, 2020

Factor geomorfológico. El modelo estructural de la superficie terrestre del Municipio Manaure, Cesar, es el resultado de diversos procesos naturales causados por movimientos tectónicos ocurridos durante miles de años, además, se encuentran afectado por los agentes exógenos actuantes en la zona como la erosión, meteorización, y el transporte de material particulado, desde las partes altas hacia las partes más bajas. Teniendo en cuenta la información obtenida de la memoria explicativa de la plancha 27 del IGAC (2019), y basándose en la corroboración realizada en campo e interpretación fotogeológica, fue posible determinar las diferentes unidades geomorfológicas (ver Tabla 3) y mapearlas (ver Figura 3) pertenecientes al área de estudio.

- Sierra denudada (Dsd): Prominencia topográfica de morfología montañosa y elongada de laderas largas a extremadamente largas, cóncavas a convexas, con pendientes muy inclinadas a abruptas, donde prevalecen procesos de erosión o de movimientos en masa acentuados.

- Abanico aluvial antiguo (Faaa): Superficie en forma de cono, de laderas cóncavas a convexas de morfología plana, aterrazada. Su origen es relacionado a la acumulación torrencial y fluvial en forma radial donde una corriente desemboca en una zona plana. Los canales fluyen cortando el abanico, siendo mas profundos en el ápice del abanico y mas someros al alejarse de él.

- Cauce aluvial (Fca): Canal de forma irregular excavado por erosión de las corrientes perennes o estacionales, dentro de macizos rocosos y/o sedimentos aluviales. Dependiendo de factores como pendiente, resistencia del lecho, carga de sedimentos y caudal, pueden persistir por grandes distancias. Los cauces rectos se restringen a valles estrechos en forma de $\mathrm{V}$.

- Espolón alto de longitud larga (Sesal): Saliente de morfología alomada, dispuesta perpendicularmente a la tendencia estructural general de la región, desarrollados sobre rocas ígneas, metamórficas o sedimentarias y limitado por drenajes paralelos a subparalelos. Con laderas de longitudes variables, y pendientes que se ven reducidas de abruptas a inclinadas por intensos procesos denudativos. 
- $\quad$ Espolón moderado de longitud larga (Sesml): Morfología alomada, desarrollada sobre rocas ígneas, metamórficas o sedimentarias y limitado por drenajes paralelos o subparalelos. Posee laderas de longitud variable con pendientes que se ven de abruptas a inclinadas por diversos procesos denudativos.

\begin{tabular}{lll} 
Unidades Geomorfológicas & Simbología & Clasificación \\
\hline Espolón alto de longitud larga & Sesal & 4 \\
\hline Sierra denudativa & Dsd & 3 \\
\hline Espolón moderado de longitud larga & Sesml & 4 \\
\hline Cauce aluvial & Fca & 1 \\
\hline Abanico aluvial antiguo & Faaa & 1 \\
\hline
\end{tabular}

Tabla 3. Unidades geomorfológicas definidas para el área de estudio Fuente: Autores, 2020
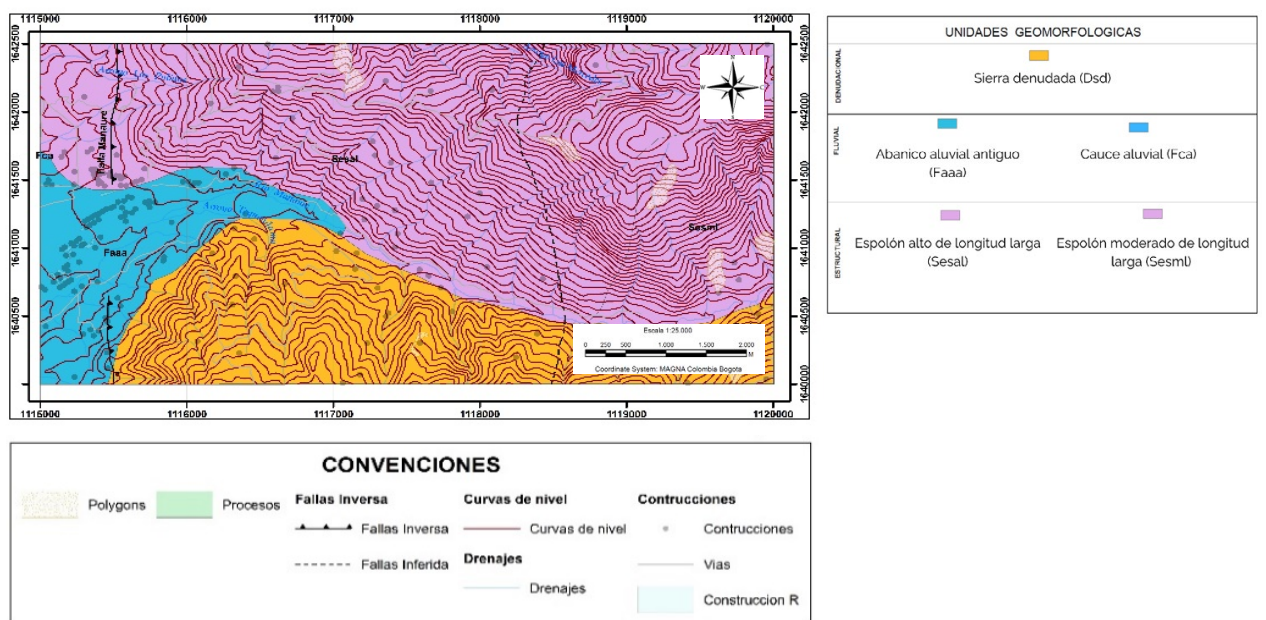

Figura 3. Mapa geomorfológico de las unidades geológicas al este de Manaure Fuente: Autores, 2020

Factor Cobertura. La efectividad de que exista cobertura vegetal favorece al terreno de mantener la estabilidad superficial y evitar su degradación constante, el que no presente cobertura vegetal, provoca que el material este expuestos a los agentes externos y del mismo modo influya de manera negativa sobre él. Teniendo en cuenta la información obtenida en campo y la interpretación fotogeológica, la cobertura vegetal fue clasificada según el tipo de vegetación y la oposición que esta presenta al momento de un fenómeno de remoción en masa (ver Tabla 4):

\begin{tabular}{ll} 
Cobertura & Calsificación \\
\hline Pastos Limpios & 5 \\
\hline Zonas Urbanas & 1 \\
\hline Bosques Fragmentados & 3 \\
\hline Bosques Densos & 1 \\
\hline Rastrojos & 1 \\
\hline
\end{tabular}

Tabla 4. Clasificación cobertura vegetal

Fuente: Autores, 2020

El siguiente mapa fue realizado con la información recolectada en campo (Tabla 4), correlacionándola con los datos del mapa de cobertura vegetal de Colombia, con lo cual obtuvimos una zona mayoritariamente poblada de vegetación secundaria, seguido de pastos y finalmente bosques naturales (ver Figura 4). 


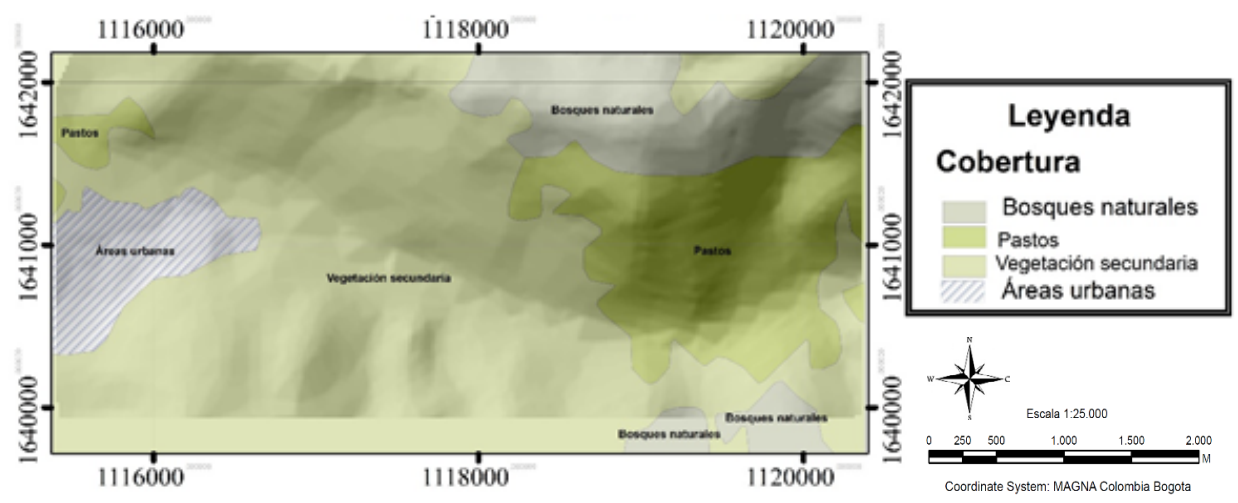

Figura 4. Mapa de cobertura vegetal del área de estudio al este de Manaure Fuente: Autores, 2020

Factor de pendiente. Para la construcción de este mapa fue necesario tener en cuenta la clasificación de pendientes que establece el Instituto Geográfico Agustín Codazzi (IGAC) siguiendo la Tabla 5:

\begin{tabular}{|c|c|c|}
\hline$\%$ & Descripción & Susceptibilidad \\
\hline $0-1 \%$ & A nivel & \multirow{3}{*}{ Muy Baja } \\
\hline $1-3 \%$ & Ligeramente plana & \\
\hline $3-7 \%$ & Ligeramente inclinada & \\
\hline $7-12 \%$ & Moderadamente inclinada & Baja \\
\hline $12-25 \%$ & Fuertemente inclinada & Media \\
\hline $25-50 \%$ & Ligeramente escarpada & Alta \\
\hline $50-75 \%$ & Moderadamente escarpada & \multirow{3}{*}{ Muy alta } \\
\hline $75-100 \%$ & Fuertemente escarpada & \\
\hline$>100 \%$ & Totalmente escarpada & \\
\hline
\end{tabular}

Tabla 5. Clasificación de pendientes

Fuente: IGAC (2019)

Esta clasificación nos permitió obtener el mapa de pendientes en la zona de estudio. Para su elaboración fueron necesarias las curvas de nivel y la utilización del software ArcGis 10.5 con algunas de sus herramientas (ver Figura 5):

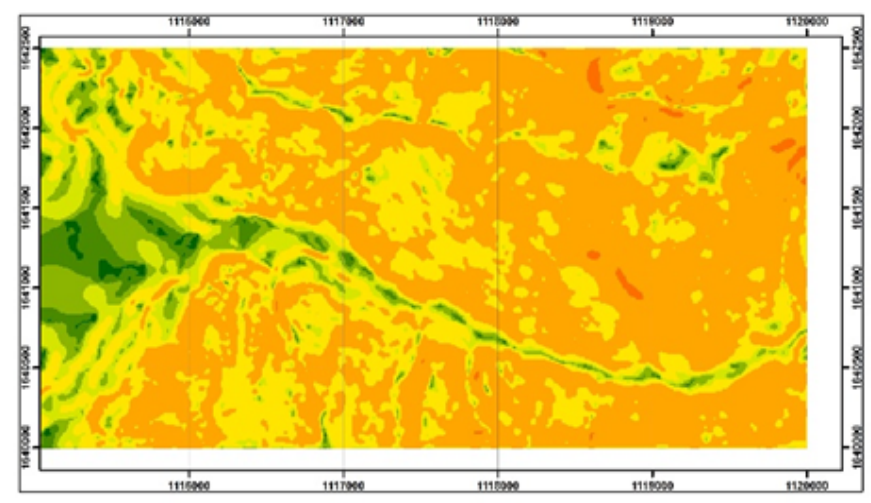

Valor de Pendiente

MUY BAJA

303

3 BAJA

3 MEDIA

3 ALTA

3 OS

MUY ALTA

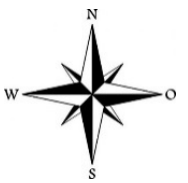

Escala 1:25.000
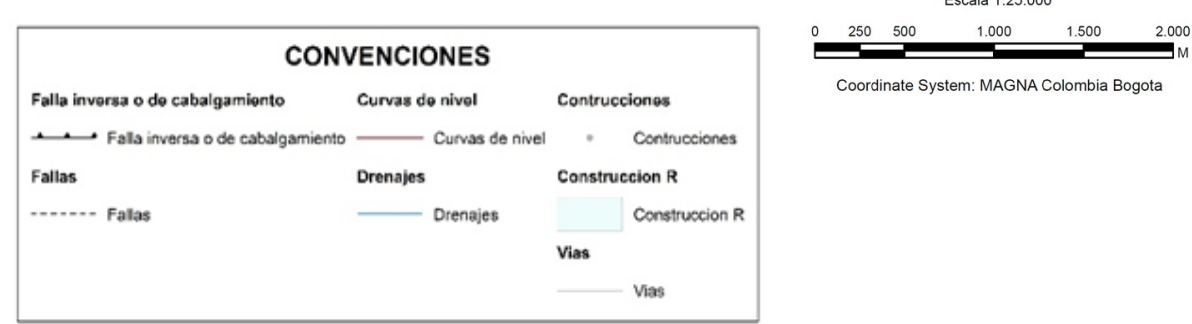

Figura 5. Mapa de porcentajes de pendientes en las unidades geológicas al este de Manaure Fuente: Autores, 2020 
Arrojando como resultados que la zona de estudio se encuentra clasificada de media a alta, ya que presenta laderas fuertemente inclinadas a ligeramente escarpadas, además, estas pendientes están condicionadas por la disposición de los macizos rocosos, por las condiciones preexistentes de inestabilidad y por la actividad sísmica, que produce aumento del esfuerzo de cizalla, la cual, favorecen a los eventos a deslizamientos de suelo y roca provocando constantes fenómenos de remoción en masa.

Al tener toda la información de los factores condicionantes o pasivos, se realiza una superposición de datos lo que nos permitió obtener el mapa final de la influencia de los factores condicionantes en la zona de estudio (Figura 6), teniendo en cuenta el porcentaje distribuido (Mora \& Vahrson, 1994) (ver Tabla 6), para cada uno de los mapas que hacen parte de estos factores:

\begin{tabular}{lll} 
Tipo & Parámetro & Porcentual (\%) \\
\hline \multirow{2}{*}{ CONDICIONANTES } & Geología & 30 \\
\cline { 2 - 3 } & Geomorfología & 20 \\
\cline { 2 - 3 } & Pendiente & 30 \\
\cline { 2 - 3 } & Cobertura vegetal & 20 \\
\hline & Total & 100 \\
\hline
\end{tabular}

Tabla 6. Valor porcentual de los factores condicionantes Fuente: Mora y Vahrson (1994)

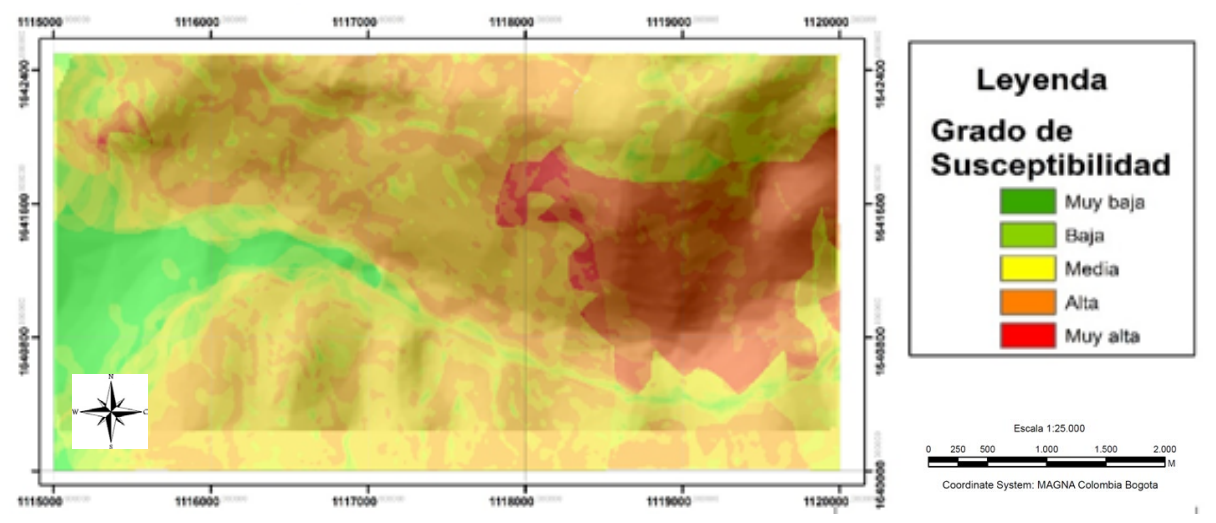

Figura 6. Mapa de factores condicionantes del área de estudio al este de Manaure Fuente: Autores, 2020

Teniendo como resultado el mapa de factores condicionantes, se puede observar cuales son las zonas con mayor grado de susceptibilidad, en este caso los colores rojizos, susceptibilidad de grado medio en las zonas donde la coloración es anaranjada y una susceptibilidad de grado bajo los colores verdes.

\section{Susceptibilidad por factores desencadenantes}

Los parámetros que corresponde a estos factores desencadenantes están constituidos por la intensidad sísmica (sismicidad) y por la intensidad de lluvias o pluviosidad (Barrantes et al., 2011).

Factor de sismicidad. La elaboración de este mapa fue posible teniendo en cuenta la información de la sismicidad de Colombia y la zona de estudio, tomada del Servicio Geológico Colombiano (SGC), teniendo en cuenta estos datos, se obtuvo como resultado el siguiente mapa (ver Figura 7):
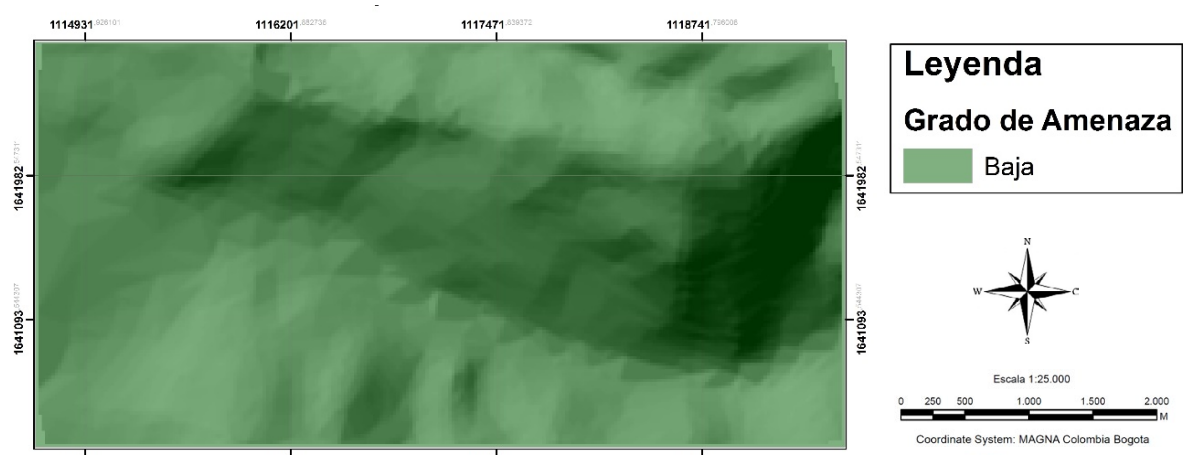

Figura 7. Mapa de sismicidad del área de estudio, al este de Manaure Fuente: Autores, 2020 
La Figura 7 nos muestra como resultado, que el área de estudio presenta una baja sismicidad, esto es debido a que no se presenta actividad de ninguna falla en el área de estudio.

Factor de pluviosidad. Para la elaboración del mapa por intensidad de lluvias se consultaron las estaciones de monitoreo de varios municipios del Departamento del Cesar y uno de la Guajira. Este segundo se tomó por su proximidad a la zona de estudio. En la Tabla 7 se identifican los $\mathrm{mm}^{3}$ de precipitaciones anuales de cada uno.

\begin{tabular}{|c|c|c|}
\hline Departamento & Municipio & Precipitación anual $\left(\mathrm{mm}^{3}\right)$ \\
\hline Cesar & Aguachica & 1416,53062 \\
\hline Cesar & Agustín Codazzi & 11240,52274 \\
\hline Cesar & Astrea & 3140,93479 \\
\hline Cesar & Becerril & 3010,84524 \\
\hline Cesar & Bosconia & 4034,05874 \\
\hline Cesar & Chimichagua & 7295,44361 \\
\hline Cesar & Chiriguaná & 4151,83196 \\
\hline Cesar & Curumaní & 8903,81716 \\
\hline Cesar & El Paso & 4204,10968 \\
\hline Cesar & Gamarra & 2778,10333 \\
\hline Cesar & La Gloria & 2123,31905 \\
\hline Cesar & La Jagua de Ibirico & 1970,12825 \\
\hline Cesar & Manaure & 1966,71333 \\
\hline Cesar & Pailitas & 3918,4628 \\
\hline Cesar & Pelaya & 1660,83846 \\
\hline Cesar & Pueblo Bello & 3519,86666 \\
\hline Cesar & Rio De Oro & 5758,17334 \\
\hline Cesar & San Alberto & 7142,79234 \\
\hline Cesar & San Diego & 5402,42708 \\
\hline Cesar & San Martin & 2604,35333 \\
\hline Cesar & Tamalameque & 3522,91689 \\
\hline Cesar & Valledupar & 16613,477316 \\
\hline Guajira & Villanueva & 1088,70458 \\
\hline
\end{tabular}

Tabla 7. Precipitaciones del Departamento del Cesar Fuente: IDEAM (2015)

Teniendo en cuenta los datos de la Tabla 7, se procedió a elaborar el mapa de precipitación del área de estudio (ver Figura 8).

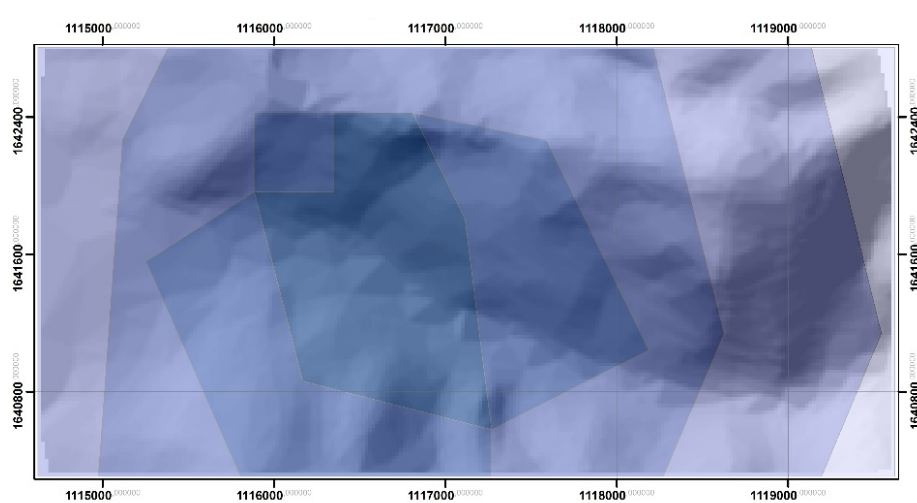

Figura 8. Mapa de precipitación correspondiente al área de estudio al este de Manaure Fuente: Autores, 2020

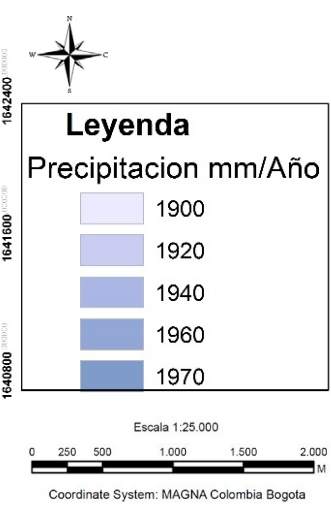


En este mapa se pueden observar las precipitaciones anuales de la zona de estudio, que van desde 1900 a $1970 \mathrm{~mm}^{3} /$ año, la cual se clasifica en un rango de 3, indicando que las precipitaciones son medias.

Al asociar los mapas de sismicidad y precipitaciones se obtiene como resultado el mapa de factores desencadenantes (ver Figura 9). Cabe resaltar que, para la obtención de estos mapas, a cada uno de los parámetros se le dio un peso porcentual y se distribuyó según Mora y Vahrson (1994) de la siguiente manera (Tabla 8).

\begin{tabular}{lll} 
Tipo & Parámetro & Porcentual (\%) \\
\hline \multirow{2}{*}{ Desencadenantes } & Precipitación & 70 \\
\cline { 2 - 3 } & Sismicidad & 30 \\
\hline & Total & 100 \\
\hline
\end{tabular}

Tabla 8. Parámetros desencadenantes

Fuente: Mora y Vahrson (1994)

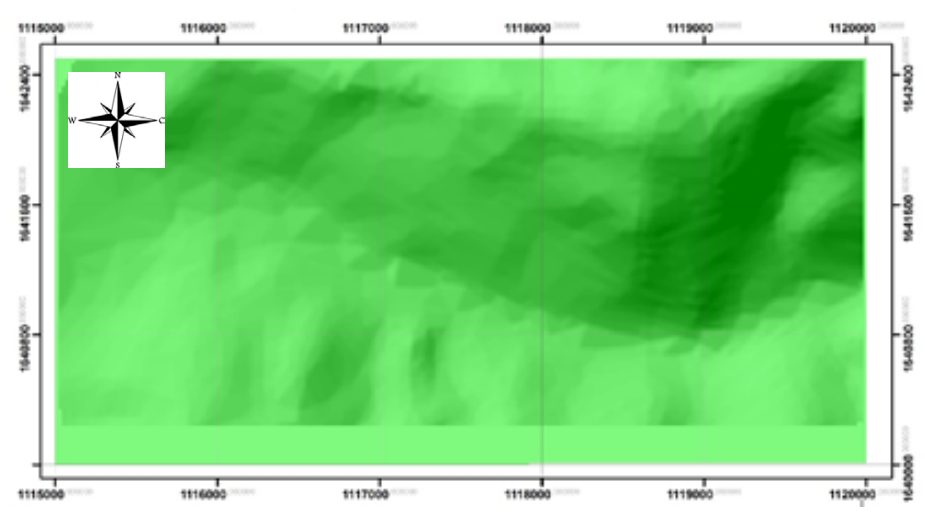

\section{Leyenda \\ Grado de Susceptibilidad}

Muy baje

$\square$ Baja

$\square$ Media

Nita

Muy alta

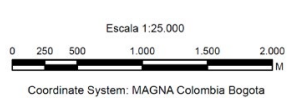

Figura 9. Mapa de factores desencadenantes correspondiente al área de estudio al este de Manaure Fuente: Autores, 2020

Posteriormente, al aplicar la fórmula de susceptibilidad (ver Ecuación 1), y correlacionarla con los datos recopilados en la tabla 8 (Barrantes et al., 2011) y se obtiene el mapa final de zonificación (ver Figura 10), el cual clasifica el área según el grado de susceptibilidad para presentar procesos de remoción en masa en el área de estudio. Como resultado se puede observar un nivel bajo en los factores desencadenantes, dado que se encontró una actividad baja de sismicidad, y los niveles de precipitaciones son intermedias, considerando que para que ocurra un deslizamiento es necesario tener un movimiento que desencadene dicho fenómeno.

Este mapa de factores condicionantes y desencadenantes es el producto de los elementos pasivos y de los factores de disparo (Barrantes et al., 2011).
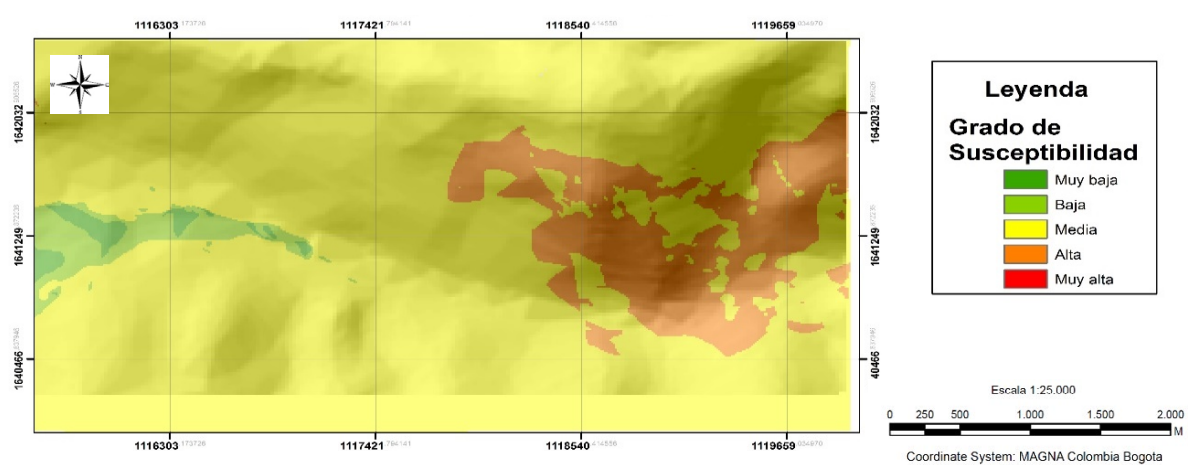

Figura 10. Mapa de zonificación correspondiente al área de estudio al este de Manaure Fuente: Autores, 2020 


\begin{tabular}{|c|c|c|}
\hline Clase & $\begin{array}{l}\text { Calificativo de } \\
\text { Susceptibilidada } \\
\text { Deslizamientos }\end{array}$ & Características \\
\hline I & Muy baja & $\begin{array}{l}\text { Sectores estables, no se requieren medidas correctivas. Se debe considerar } \\
\text { la influencia de los sectores aledaños con susceptibilidad de moderada a muy } \\
\text { alta. Sectores aptos para usos urbanos de alta densidad y ubicación de edificios } \\
\text { indispensables como hospitales, centros educativos, estaciones de policía, bomberos, } \\
\text { etc. }\end{array}$ \\
\hline ॥ & Baja & $\begin{array}{l}\text { Sectores estables que requieren medidas correctivas menores, solamente en caso de } \\
\text { obras de infraestructura de gran envergadura. Se debe considerar la influencia de los } \\
\text { sectores aledaños con susceptibilidad de moderada a muy alta. Sectores aptos para } \\
\text { usos urbanos de alta densidad y ubicación de edificios indispensables como hospitales, } \\
\text { centros educativos, estaciones de policía, bomberos, etc. Los sectores con rellenos mal } \\
\text { compactados son de especial cuidado. }\end{array}$ \\
\hline III & Moderada & $\begin{array}{l}\text { No se recomienda la construcción de infraestructura si no se realizan estudios } \\
\text { geotécnicos y se mejora la condición del sitio. Las mejoras pueden incluir: movimientos } \\
\text { de tierra, estructuras de retención, manejo de aguas superficiales y subterráneas, } \\
\text { reforestación, entre otros. Los sectores con rellenos mal compactados son de especial } \\
\text { cuidado. Recomendable para usos agropecuario. }\end{array}$ \\
\hline IV & Alta & $\begin{array}{l}\text { Probabilidad de deslizamiento alta }(<50 \%) \text { en caso de sismos de magnitud importante } \\
\text { y lluvias de intensidad alta. Para su utilización se deben realizar estudios estabilidad } \\
\text { a detalle y la implementación de medidas correctivas que aseguren la estabilidad del } \\
\text { sector, en caso contrario, deben mantenerse como áreas de protección. }\end{array}$ \\
\hline
\end{tabular}

Tabla 9. Clasificación de la susceptibilidad a deslizamientos

Fuente: Mora (2004)

\section{DISCUSIÓN}

Resultados de factores desencadenantes

En la zona de estudio según la revisión bibliográfica realizada a los documentos de precipitaciones medias anuales entregados por el IDEAM (2015) y Quesada y Feoli (2018), se determinó que el área presenta unas precipitaciones anuales de 1900-1970 mm³ lo cual la ubica en una clasificación media. Por otro lado, teniendo los resultados de la sismicidad del área (ver Figura 7), estos nos muestran que es baja, ya que la falla Manaure se encuentra inactiva. Al evaluar el escenario para que se presente un evento de remoción en masa es necesario tener una relación de similitud entre las precipitaciones y la sismicidad, sin embargo, no existen registros que estos fenómenos de remoción sean frecuentes en el área de estudio.

Según el método empleado, la zona este presenta un grado de susceptibilidad a movimientos en masa medio y bajo, mientras que la zona de montaña, hacia el oeste presenta en ciertas partes grados altos de susceptibilidad. Sin embargo, el casco urbano del municipio presenta susceptibilidad baja a deslizamientos. Por lo anterior, es importante tener en cuenta estas zonas al momento de realizar planificaciones en el área urbana o en zonas aledañas a esta.

\section{Hipótesis dado el caso que la falla Manaure llegase a activarse}

En caso de que la falla se activara, los índices de susceptibilidad aumentarían de manera exponencial, dado que, para que haya un alto grado, según el método de estudio, ambos parámetros (condicionantes y desencadenantes) deben tener valores semejantes (ver Figura 11). En tal caso, según la hipótesis que se manejó, se tendría un grado de susceptibilidad alto en el $90 \%$ del área, los cuales podrían generar movimientos de remoción en masa.

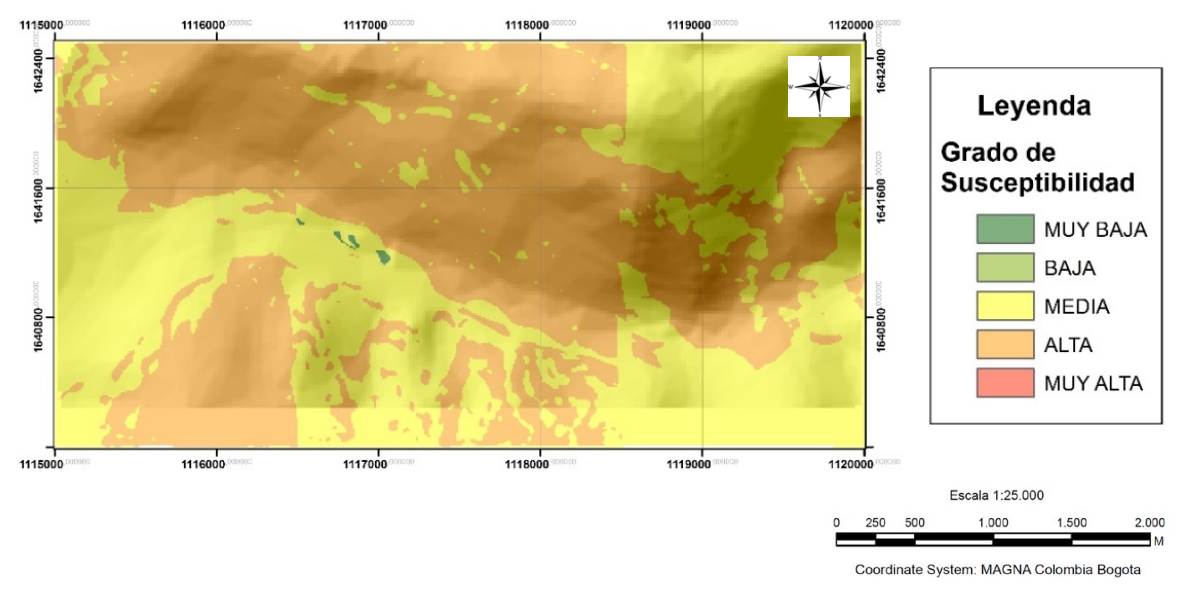


Sin embargo, no es despreciable el $10 \%$ restante que tendría una susceptibilidad media, esto incluye el casco urbano del municipio. Con lo anterior, se observa la necesidad de la realización de más estudios para explorar ésta y otras hipótesis.

\section{CONCLUSIONES}

La zonificación de la susceptibilidad a movimientos en masa en el municipio de Manaure muestra, de acuerdo con la metodología empleada, valores intermedios y bajos, apoyando los datos históricos de centro poblado donde no se conocen registros de este tipo de movimientos a pesar de tener una geomorfología de pendientes medias y altas. Su baja susceptibilidad se debe principalmente al poco actuar de los factores desencadenantes además de los tipos de litologías del área dentro de los factores condicionantes. Sin embargo, en un escenario de reactivación de la falla Manaure tendríamos una zona con susceptibilidad alta en la mayor parte del territorio, lo que implicaría toma de medidas preventivas para sus habitantes y los gobiernos locales.

Otro parámetro muy importante en el análisis fue la precipitación, puesto que las rocas metasedimentarias según el grado de humedad son las que pueden presentan mayor grado de susceptibilidad a la remoción en masa. Basándonos en los parámetros evaluados en condiciones normales la posibilidad de ocurrencia (aunque sea baja) de fenómenos, estos indican que las remociones en masa en la zona de estudio tenderían a ser producto de las precipitaciones ocurridas, más no de la sismicidad de la zona.

Finalmente, el análisis realizado en la zona y el funcionamiento de la metodología implementada sugiere tener en cuenta los siguientes aspectos en futuros estudios.

- Realizar estudios geológico-geotécnicos más detallados en el área de estudio para poder determinar los métodos de prevención a los fenómenos que se puedan presentar.

- Analizar dentro del plan de ordenamiento territorial del municipio la influencia de la zona de la falla Manaure. Y si ella llegara a activarse, considerar que existen registros de movimientos telúricos en zonas cercanas al municipio.

- Aplicar métodos de estabilidad en las áreas que presenten una mayor inestabilidad, debido a las precipitaciones de la zona.

\section{REFERENCIAS}

Acosta, C. \& Rodríguez, Y. (2016). Identificación y caracterización de escenarios de riesgo en zona urbana del municipio de Manaure y sus veredas, en el Departamento del Cesar. Valledupar, Colombia: Fundación Universitaria del Área Andina.

Campos, A., Holm-Nielsen, N., Díaz, C., Rubiano, D.M., Costa, C.R., Ramírez, F. \& Dickson, E. (2012). Análisis de la gestión del riesgo de desastres en Colombia. Bogotá: Banco Mundial \& Banco Internacional de Reconstrucción y Fomento. Recuperado de: http://hdl.handle. net $/ 20.500 .11762 / 18426$

Barrantes Castillo, G., Barrantes Sotela, O. \& Nuñes Román, O. (2011). Efectividad de la metodología Mora-Vahrson modificada en el caso de los deslizamientos provocados por el terremoto de Cinchona, Costa Rica. Revista Geográfica de América Central, 42(2), 141-162.

Gobernación del Cesar. (2019). Departamento del Cesar. Gobernación del Cesar. Recuperado de: http:// cesar.gov.co/d/index.php/es/mainmeneldpto/mendeppre

González, L., Ferrer, M., Ortuño, L. \& Oteo, C. (2002). Ingeniería Geológica. Madrid: Pearson Educación.

Gutierrez, M. (2008). Geomorfologia. Madrid: Pearson Educación.

Hidalgo, C. \& Vega, J. (2014). Estimación de la Amenaza por Deslizamientos Detonados por Sismos y Lluvia (Valle de Aburrá, Colombia). Revista EIA, 11(22), 103-117.

IDEAM. (2015). Precipitaciones Mensuales, Anuales de Colombia. Bogotá: IDEAM

IGAC. (2019). Plancha 27. Instituto Geográfico Agustín Codazzi. Recuperado de: https://geoportal.igac. gov.co/contenido/consulta-de-planchas

Mora, R. (2004). Evaluación de la Susceptibilidad al Deslizamiento del Cantón de San José, Provincia de San José, Costa Rica. San José: Escuela Centroamericana de Geología.

Mora, S. \& Vahrson, W. (1994). Macrozonation methodology for landslide hazard determination. Bulleting of the Association of Engineering and Geologist, 31(1), 49-58. 
Quesada, A. \& Feoli, S. (2018). Comparación de la Metodología Mora-Vahrson y el Método Morfométrico para Determinar Âreas Susceptibles a Deslizamientos en la Microcuenca del Río Macho, Costa Rica. Revista Geográfica de América Central, 61(2), 17-45. http://dx.doi.org/10.15359/ rgac.61-2.1

Ramírez, M. (2006). Evaluación del Peligro por fenómenos de remoción en masa y su aplicación a la planificación territorial. Quito: Escuela Politécnica Nacional.

Suares, J. (1998). Deslizamientos Y Estabilidad De Taludes En Zonas Tropicales. Bucaramanga: Instituto de Investigaciones sobre Erosión y Deslizamientos.

Vivanco, C. (2011). Análisis Comparativo De Técnicas Estadísticas Y De Aprendizaje Para Evaluar La Susceptibilidad Del Terreno A Los Deslizamientos Superficiales. Loja, Ecuador: Universidad Técnica Particular de Loja. 